\title{
Parameterisation of the residual temperature distribution based on the modelling of successive emission of prompt neutrons
}

\author{
Anabella Tudora ${ }^{1, \star}$ and Franz-Josef Hambsch ${ }^{2}$ \\ ${ }^{1}$ University of Bucharest, Faculty of Physics, 405 Atomistilor Str., Magurele 077125, Romania \\ ${ }^{2}$ E.C. Joint Research Centre, Directorate G, Unit G.2., Retieseweg 111, 2440 Geel, Belgium
}

\begin{abstract}
A new deterministic modelling taking into account the successive emission of prompt neutrons from initial fragments of a fragmentation range $\{\mathrm{A}, \mathrm{Z}, \mathrm{TKE}\}$ constructed as in the Point-by-Point $(\mathrm{PbP})$ treatment is described. The good agreement of different prompt emission quantities obtained from this modelling (e.g. $v(\mathrm{~A}), v(\mathrm{TKE})$, $\mathrm{E} \gamma(\mathrm{A}), \mathrm{E} \gamma(\mathrm{TKE})$, etc.) with the experimental data and the results of the PbP model and other Monte-Carlo models validates the present modelling of sequential emission. The distributions of different residual quantities, including the residual temperature distributions $\mathrm{P}(\mathrm{T})$ of light and heavy fragments allow to obtain a new parameterisation of $\mathrm{P}(\mathrm{T})$ which can be used in the PbP model and the Los Alamos model.
\end{abstract}

\section{Introduction}

This paper reports preliminary results of a deterministic treatment of the successive emission of prompt neutrons. The modelling starts with the construction of the initial fragmentation range as in the Point-by-Point $(\mathrm{PbP})$ model of prompt emission [1]. For each initial fragment $\mathrm{A}, \mathrm{Z}$ at a given TKE covering the range of (A,Z,TKE) an equation for the residual temperature can be solved for each sequence of successive neutron emission. Then different quantities characterizing the residual nucleus and the prompt emission are obtained for each emission sequence (indexed $\mathrm{k}$ ) corresponding to an initial fragment $\mathrm{A}, \mathrm{Z}$ at a given TKE, where $\mathrm{k}$ is running over the number of prompt neutrons emitted successively (or the number of residual fragments). Using the multiple distributions Y(A,Z,TKE) of initial fragments, different distributions, e.g. of the residual temperature, the residual energy, the average neutron energy in the centre-of-mass frame corresponding to the emission of each neutron as well as the sum of these distributions following the emission of all neutrons can be obtained. Different quantities generically labelled $\mathrm{q}(\mathrm{A}, \mathrm{Z}, \mathrm{TKE})$ corresponding to an initial fragment at a TKE value (e.g. the prompt neutron multiplicity $v(\mathrm{~A}, \mathrm{Z}, \mathrm{TKE})$, the average prompt $\gamma$-ray energy $\mathrm{E} \gamma(\mathrm{A}, \mathrm{Z}, \mathrm{TKE})$, the prompt neutron spectrum in the centre-of-mass frame $\varphi(\varepsilon, \mathrm{A}, \mathrm{Z}, \mathrm{TKE})$ etc.) can be obtained by averaging the respective quantities corresponding to each sequence $\mathrm{q}_{\mathrm{k}}(\mathrm{A}, \mathrm{Z}, \mathrm{TKE})$ over the number of sequences (i.e. the number of emitted neutrons).

This modelling was applied to three fissioning nuclei, i.e. ${ }^{252} \mathrm{Cf}(\mathrm{SF})$ and ${ }^{235} \mathrm{U}\left(\mathrm{n}_{\mathrm{th}}, \mathrm{f}\right)$ for which $\mathrm{Y}(\mathrm{A}, \mathrm{TKE})$ distributions and prompt neutron data were recently measured at JRC-Geel $[2,3]$ and ${ }^{239} \mathrm{Pu}\left(\mathrm{n}_{\mathrm{th}}, \mathrm{f}\right)$ for which $\mathrm{Y}(\mathrm{A}, \mathrm{TKE})$ data measured at JRC-Geel and prompt emission data are available

^e-mail: anabellatudora@hotmail.com 
in the EXFOR library. The good agreement of different prompt emission quantities obtained from this modelling (e.g. $v(\mathrm{~A}), v(\mathrm{TKE}), \mathrm{E} \gamma(\mathrm{A}),<\varepsilon>(\mathrm{A})$ etc.) with the experimental data and the results of other prompt emission models (e.g. PbP, FIFRELIN, etc.) validates the present deterministic modelling of the successive emission of prompt neutrons. The residual temperature distributions $\mathrm{P}(\mathrm{T})$ for the light and heavy fragment groups resulting from this modelling offer the possibility to obtain a preliminary parameterisation of $\mathrm{P}(\mathrm{T})$. This parameterisation can be used in the PbP model and the Los Alamos model where the sequential emission is taken into account in a global way by a $\mathrm{P}(\mathrm{T})$ distribution.

\section{Equation of residual temperature following the successive emission of each neutron}

For a given fissioning nucleus the initial fragmentation range is constructed as in the $\mathrm{PbP}$ treatment [1], i.e. the mass number $A$ of initial fragments covers a large range, from symmetric fission up to a very asymmetric split with a step of one mass unit. For each $\mathrm{A}$ three charge numbers $\mathrm{Z}$ are taken as the nearest integer values above and below the most probable charge taken as the unchanged charge distribution corrected with the charge polarization $\mathrm{Zp}(\mathrm{A})=\mathrm{Z}_{\mathrm{UCD}}(\mathrm{A})+\Delta \mathrm{Z}(\mathrm{A})$. The charge polarization $\Delta \mathrm{Z}(\mathrm{A})$ and the $\mathrm{rms}(\mathrm{A})$ of the isobaric charge distribution given by the $\mathrm{Zp}$ model are used [4]. For each fragmentation TKE values covering a large range (e.g. from 100 to $200 \mathrm{MeV}$ ) with a step of $5 \mathrm{MeV}$ are considered.

For each initial fragment $\mathrm{A}, \mathrm{Z}$ at each TKE (covering the fragmentation range mentioned above) a number of $k_{\max }$ prompt neutrons can be successively emitted if the excitation energy of the last residual fragment (with the charge number $\mathrm{Z}$ and the mass number $\mathrm{A}-\mathrm{k}_{\max }$ ) becomes lower than the neutron separation energy from this nucleus $\mathrm{Sn}^{(\mathrm{kmax})}$.

Considering the fragment level densities in the Fermi-gas regime, the temperature of the k-th residual nucleus (following the emission of the k-th prompt neutron) corresponding to each initial fragment at each TKE, is the solution of the following equation:

$$
{\overline{E_{r}}}^{(k-1)}-S_{n}^{(k-1)}-<\varepsilon>_{k}=a_{k} T_{k}^{2}
$$

in which ${\overline{E_{r}}}^{(k-1)}$ and $S_{n}^{(k-1)}$ are the average excitation energy and the neutron separation energy of the precursor, respectively. $\langle\varepsilon\rangle_{k}$ is the average energy of the k-th emitted neutron in the center-of-mass frame, $a_{k}$ and $T_{k}$ are the level density parameter and the temperature of the k-th residual nucleus, respectively. For the first emitted prompt neutron, $\mathrm{k}=1,{\overline{E_{r}}}^{(0)}=E^{*}$ is the excitation energy of the initial fragment (before the first neutron is emitted) resulting from the TXE partition by modelling at scission $[5,6]$.

To solve the residual temperature equations (1) the following approximations are needed:

(a) non-energy dependent level density parameters of initial and residual fragments, e.g. provided by the Egidy-Bucurescu systematic for the back-shift Fermi-gas (BSFG) model [7] or by the GilbertCameron systematic for spherical nuclei [8] and

(b) an analytical expression of the compound nucleus cross-section $\sigma_{c}(\varepsilon)$ of the inverse process of neutron evaporation which can approximate $\sigma_{c}(\varepsilon)$ provided by optical model calculations with optical potential parameterisations appropriate for nuclei appearing as fission fragments. Note, the simplest approximation is to consider a constant $\sigma_{c}$. In this case the average prompt neutron energy is $\langle\varepsilon\rangle_{k}$ $\left(T_{k}\right)=2 T_{k}$ and the residual temperature equation (1) has an analytical solution.

In the present calculations $\sigma_{c}(\varepsilon)$ of each residual nucleus is approximated according to Ref. [9] by the following expression:

$$
\sigma_{c}^{(k)}(\varepsilon)=\sigma_{0}^{(k)}\left(1+\alpha_{k} / \sqrt{\varepsilon}\right)
$$


with $\sigma_{0}^{(k)}$ and $\alpha_{k}=\left(\hbar^{2} / m r_{0}^{2}\right) S_{0}^{(k)} / A_{k}^{2 / 3}$ depending on the mass number $A_{k}$ and the s-wave neutron strength function of each residual nucleus $\mathrm{k}$.

To verify if the approximation of $\sigma_{c}(\varepsilon)$ by Eq. (2) is appropriate to be used in the calculation of $\langle\varepsilon\rangle_{k}$ entering the transcendent equations (1), the average prompt neutron energy in the centre-of-mass frame obtained with $\sigma_{c}(\varepsilon)$ of Eq. (2), i.e.

$$
<\varepsilon>(T)=T(2 \sqrt{T}+(3 \sqrt{\pi} / 4) \alpha) /(\sqrt{T}+\alpha \sqrt{\pi} / 2)
$$

was compared with $<\varepsilon>$ obtained by using $\sigma_{c}(\varepsilon)$ from optical model calculations, i.e.

$$
<\varepsilon>(T)=\int_{0}^{\infty} K(T) \varepsilon^{2} \sigma_{c}(\varepsilon) \exp (-\varepsilon / T) d \varepsilon, K(T)^{-1}=\int_{0}^{\infty} \varepsilon \sigma_{c}(\varepsilon) \exp (-\varepsilon / T) d \varepsilon
$$

The average prompt neutron energies $<\varepsilon>$ were calculated according to Eqs. (3) and (4) for temperatures ranging from $0.2 \mathrm{MeV}$ to $2 \mathrm{MeV}$ (which is the usual range of residual temperatures). The calculations were done for many nuclei covering the range of fission fragments. The deviation of $<\varepsilon>$ (T) given by Eq. (3) from $\left\langle\varepsilon>\right.$ (T) based on $\sigma_{c}(\varepsilon)$ from optical model calculations (with the Becchetti-Greenlees potential) is less than $4 \%$ for all fragments. This fact supports the use of the $\sigma_{c}(\varepsilon)$ given by Eq. (2) in the transcendent equations of residual temperatures.

In the PbP model and other refined models of prompt emission, energy-dependent level density parameters of fragments are used. They are given by the super-fluid model with different shell corrections and different parameterisations of the damping of shell effects and the asymptotic value of the level density parameter. To see if non-energy dependent level density parameters can be used in the residual temperature equations, the variation with energy of the super-fluid level density parameter of many nuclei appearing as fission fragments, for energies ranging up to about $30 \mathrm{MeV}$, was compared with the constant level density parameter provided by the BSFG systematic of Ref. [7] and the Gilbert-Cameron systematic [8]. The level density parameters of Ref. [7] were used in the present calculations because they approximate well the super-fluid level density parameters for the majority of fission fragments, except the heavy fragments with A around 130 for which the large negative values of the shell corrections (due to the magic numbers $N=82, \mathrm{Z}=50$ ) lead to a pronounced variation with energy of the super-fluid level density parameters.

Under the approximations (a) and (b) discussed above, for each initial fragment A,Z at each TKE, appearing with a probability given by the fragment distribution Y(A,Z,TKE), the iterative transcendent equations of residual temperatures given by Eq. (1), with $\mathrm{k}$ from 1 to $\mathrm{k}_{\max }(\mathrm{A}, \mathrm{Z}, \mathrm{TKE})$, are numerically solved, giving the residual temperatures $\mathrm{T}_{\mathrm{k}}(\mathrm{A}, \mathrm{Z}, \mathrm{TKE})$. The energy of each residual nucleus $E_{r}^{(k)}(\mathrm{A}, \mathrm{Z}, \mathrm{TKE})$ and the average energy in the centre-of-mass frame $<\varepsilon>_{k}(\mathrm{~A}, \mathrm{Z}, \mathrm{TKE})$ of each k neutron emitted sequentially are easily obtained, too.

Examples of distributions of residual temperature and residual energy following the successive emission of neutrons from the heavy and light fragments are illustrated in Fig. 1. The average values of the residual temperatures and residual energies, corresponding to each neutron successively emitted, are given in each frame. Note, the distributions of the last emitted neutrons $(\mathrm{k}=6,7)$, being very low, were not plotted. Any quantity (generically labelled "q") corresponding to each emitted neutron as a function of initial fragment mass A, or initial TKE can be obtained by averaging $q_{k}(\mathrm{~A}, Z, \mathrm{TKE})$ over the $\mathrm{Y}(\mathrm{A}, \mathrm{Z}, \mathrm{TKE})$ distribution, by summing over $\mathrm{Z}$ and TKE and over $\mathrm{A}$ and $\mathrm{Z}$, respectively. An example of average residual temperatures and energies following the successive emission of each neutron as a function of initial fragment mass A is illustrated in Fig. 2. The saw-tooth shape of these quantities corresponding to the first, second and third emitted neutron is visible. As it can be seen, the successive emission of more than 3 neutrons (i.e. $\mathrm{k}=4,5,6,7$ ) is not possible for all initial fragment masses. 

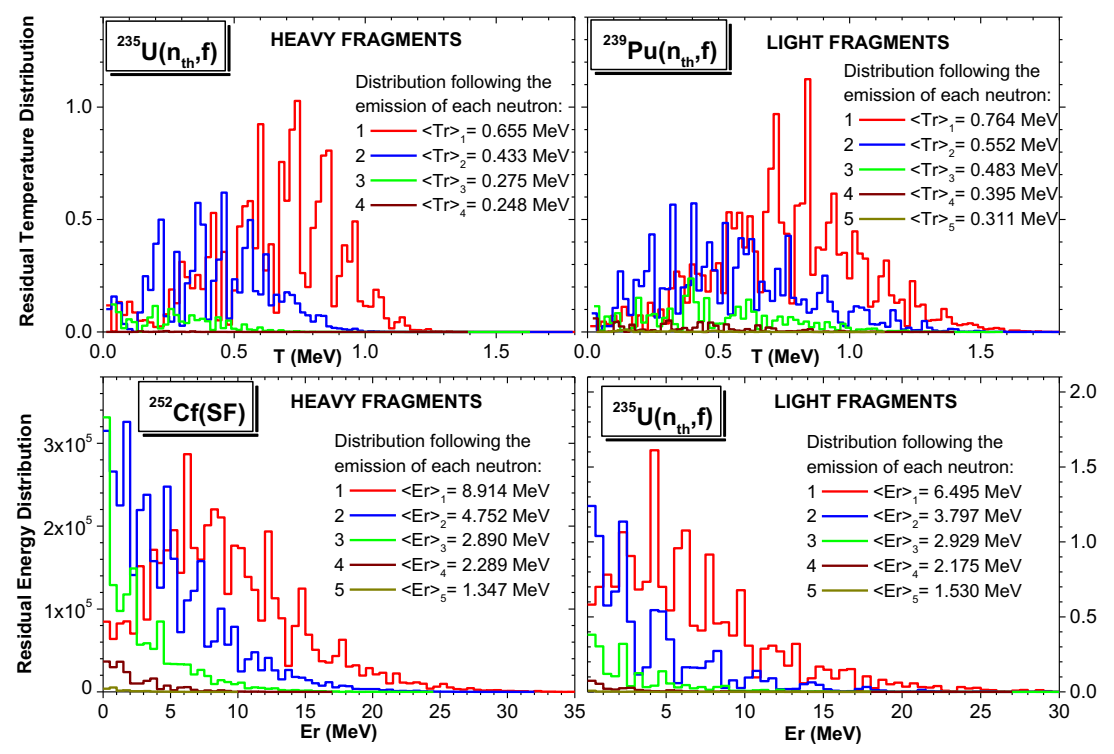

Figure 1. Examples of residual temperature and residual energy distributions following the emission of the first (red lines), second (blue lines), third (green lines), 4-th (wine line) and 5-th neutron (dark yellow) from the heavy and light fragments.
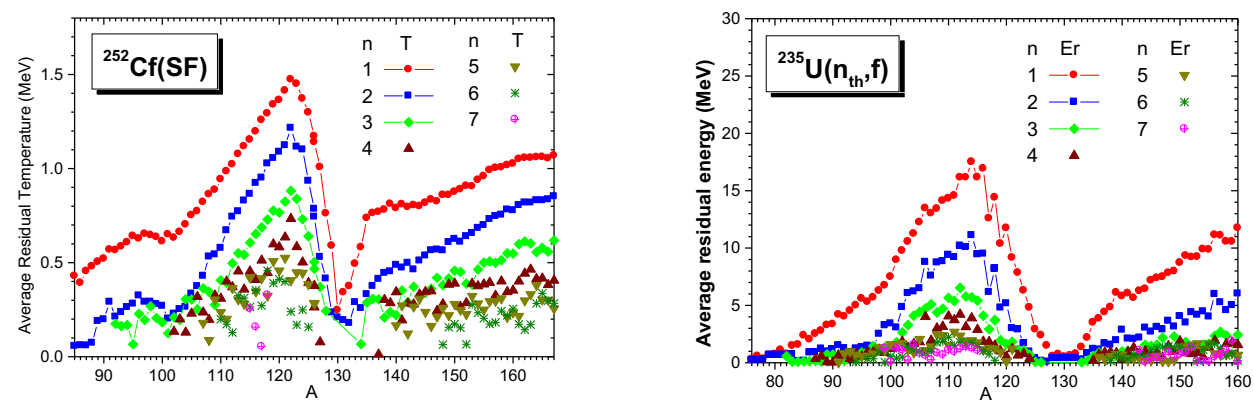

Figure 2. Average residual temperatures (left part) and average residual energies (right part) following the successive emission of each neutron as a function of initial fragment mass A, exemplified for ${ }^{252} \mathrm{Cf}(\mathrm{SF})$ and ${ }^{235} \mathrm{U}\left(\mathrm{n}_{\mathrm{th}}, \mathrm{f}\right)$, respectively.

\section{Validation of sequential emission calculation by comparison with experimental data and results of other prompt emission models}

Any prompt emission quantity corresponding to an initial fragment $A, Z$ at a TKE value, q(A,Z,TKE), can be obtained by averaging $q_{k}(A, Z, T K E)$ over the number of sequential emissions $k_{\max }(A, Z, T K E)$, i.e.:

$$
q(A, Z, T K E)=\frac{1}{k_{\max }(A, Z, T K E)} \sum_{k=1}^{K \max } q_{k}(A, Z, T K E)
$$




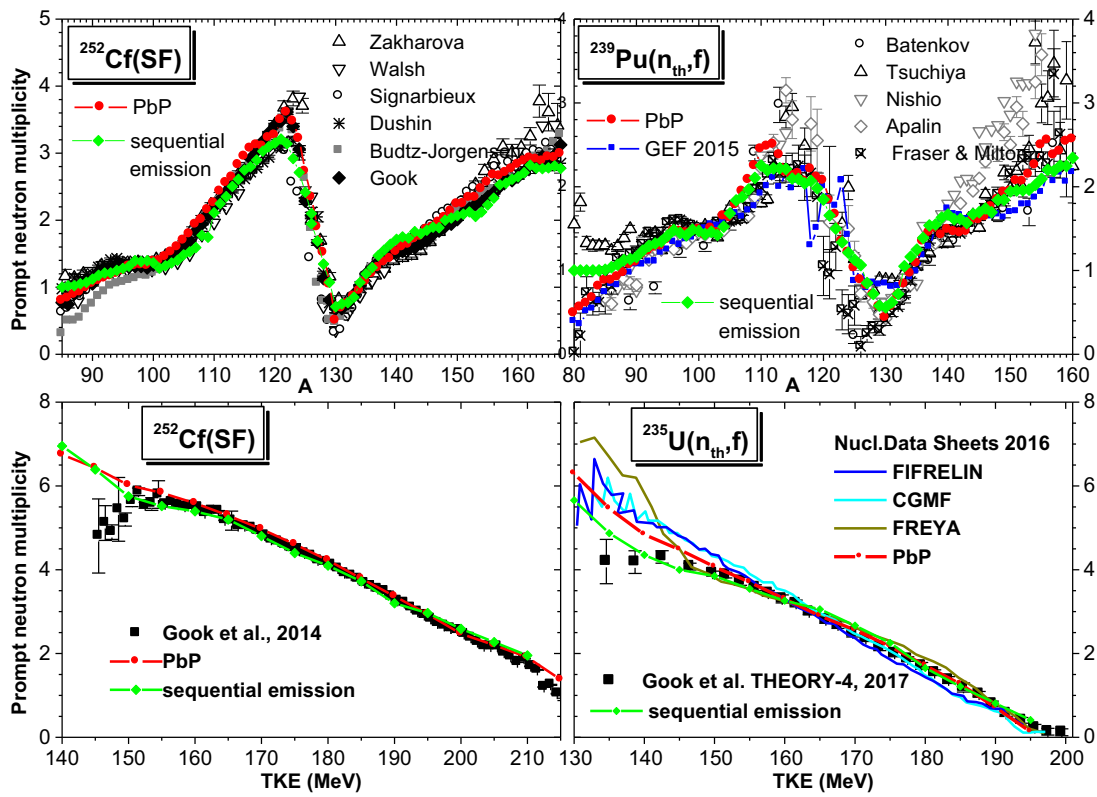

Figure 3. Examples of $v(\mathrm{~A})$ and $\langle v>$ (TKE) resulting from sequential emission calculations (green diamonds) in comparison with experimental data (full and open black and grey symbols), the PbP result (red circles) and the results of other prompt emission models.

Different average prompt emission quantities, as a function of initial mass $\mathrm{A}, \bar{q}(\mathrm{~A})$, as a function of TKE, $<q>$ (TKE) are then obtained by averaging $q(A, Z, T K E)$ of Eq. (5) over the Y(A,Z,TKE) distribution (by summing over $Z$ and TKE and over $A$ and $Z$, respectively). These average quantities are compared with the existing experimental data and also with the results of other prompt emission models. Examples of such comparisons are given in Figs. 3 and 4.

The $v(\mathrm{~A})$ results of sequential emission calculation (green diamonds, upper part of Fig. 3) describe well the experimental data. They are close to the $\mathrm{PbP}$ results (red circles) and exhibit a more pronounced structure compared to $v(\mathrm{~A})$ of $\mathrm{PbP}$.

As it can be seen in the lower part of Fig. 3 the PbP results of $\langle v\rangle$ (TKE) (red circles connected with lines) give an excellent description of the experimental data of Göök et al. of ${ }^{252} \mathrm{Cf}(\mathrm{SF})$ [2] and especially of ${ }^{235} \mathrm{U}\left(\mathrm{n}_{\mathrm{th}}, \mathrm{f}\right)$ (this workshop [10]). Note, the PbP result of $\left\langle v>\right.$ (TKE) for ${ }^{235} \mathrm{U}\left(\mathrm{n}_{\mathrm{th}}, \mathrm{f}\right)$ as well as the results of the computer codes FIFRELIN (blue line), CGMF (cyan line) and FREYA (dark yellow line) were predictions, being reported at the beginning of 2016 [1], before the experimental data of Göök et al. (preliminary results reported in September 2016 [3] and the results presented at this workshop). The $\langle v\rangle$ (TKE) results of sequential emission (green diamonds connected with lines) are also in very good agreement with the experimental data and the results of $\mathrm{PbP}$.

Examples of $\mathrm{E} \gamma(\mathrm{A})$ resulting from sequential emission calculations for ${ }^{235} \mathrm{U}\left(\mathrm{n}_{\mathrm{th}}, \mathrm{f}\right)$ and ${ }^{239} \mathrm{Pu}\left(\mathrm{n}_{\mathrm{th}}, \mathrm{f}\right)$ are plotted in the upper part of Fig. 4 with green diamonds in comparison with the experimental data of Pleasonton et al. (black squares) and the results of the PbP model (red circles). Significant differences between the results of $\mathrm{PbP}$ and the sequential emission calculation are visible near symmetric fission (where experimental data are missing). Both calculations are in reasonable agreement with the scattered experimental data. 


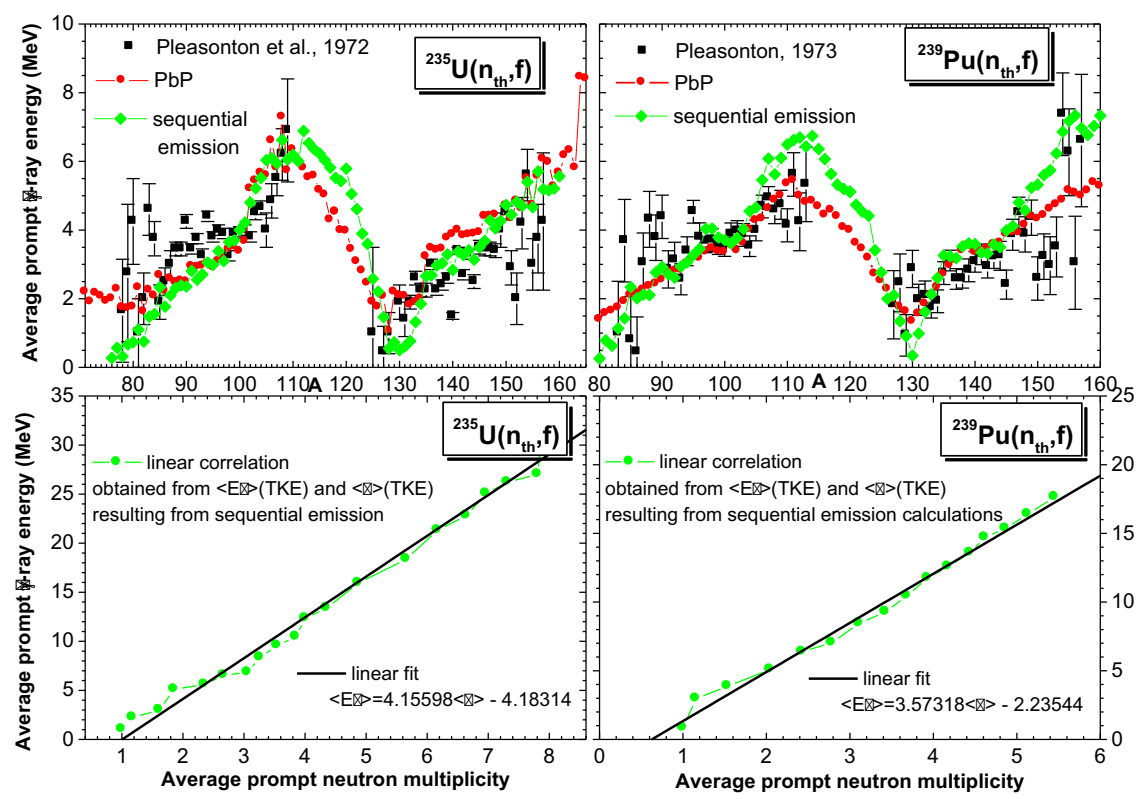

Figure 4. Upper part: $\mathrm{E} \gamma(\mathrm{A})$ of ${ }^{235} \mathrm{U}\left(\mathrm{n}_{\mathrm{th}}, \mathrm{f}\right)$ and ${ }^{239} \mathrm{Pu}\left(\mathrm{n}_{\mathrm{th}}, \mathrm{f}\right)$ : the sequential emission result (green diamonds) in comparison with the experimental data of Pleasonton et al. (black squares) and the PbP result (red circles). Lower part: the linear correlation between the prompt $\gamma$-ray energy and the prompt neutron multiplicity (green circles) obtained from $\langle\mathrm{E} \gamma\rangle$ (TKE) and $\langle v\rangle$ (TKE) resulting from sequential emission calculation and the linear fit (black line).

The sequential emission calculations confirm the linear correlation between the average prompt $\gamma$-ray energy and the prompt neutron multiplicity, as it can be seen in the lower part of Fig. 4 where the correlation resulting from the sequential emission results of $\langle\mathrm{E} \gamma>$ (TKE) and $\langle v>$ (TKE) is plotted with green circles and the corresponding liner fit with a black line.

As it can seen in the upper parts of Figs. 3 and 4,v(A) and $\mathrm{E} \gamma(\mathrm{A})$ resulting from sequential emission calculations exhibit a more pronounced structure than the $\mathrm{PbP}$ results. This fact is not surprising because the modelling of sequential emission is a detailed treatment of the emission of each neutron from each residual fragment formed successively, while the PbP model takes globally into account the sequential emission just by a $\mathrm{P}(\mathrm{T})$ distribution with a smooth form. An example is the prompt $\gamma$-ray energy corresponding to each initial fragment $\mathrm{A}, \mathrm{Z}$ at a given TKE. This is calculated in the $\mathrm{PbP}$ model as

$$
E_{\gamma}(A, Z, T K E)=E^{*}(A, Z, T K E)-v(A, Z, T K E)(<\varepsilon>(A, Z, T K E)+\bar{S} n(A, Z, T K E))
$$

in which $E^{*}(A, Z, T K E)$ is the excitation energy of the initial fragment (resulting from the TXE partition by modelling at scission), $v(A, Z, T K E)$ is the prompt neutron multiplicity, the neutron separation energy is an average value calculated as $S_{k n} / k$ (with k the number of prompt neutrons emitted sequentially). The first order momentum of the prompt neutron spectrum in the centre-of-mass frame $\langle\varepsilon>$ entering Eq. (6) is obtained by averaging the evaporation spectrum at a given residual temperature over a residual temperature distribution $P(T)$ with a smooth form. In the sequential emission treatment $\mathrm{E} \gamma$ is calculated at each emission sequence $\mathrm{k}$ corresponding to an initial fragment $\mathrm{A}, \mathrm{Z}$, at a given TKE 

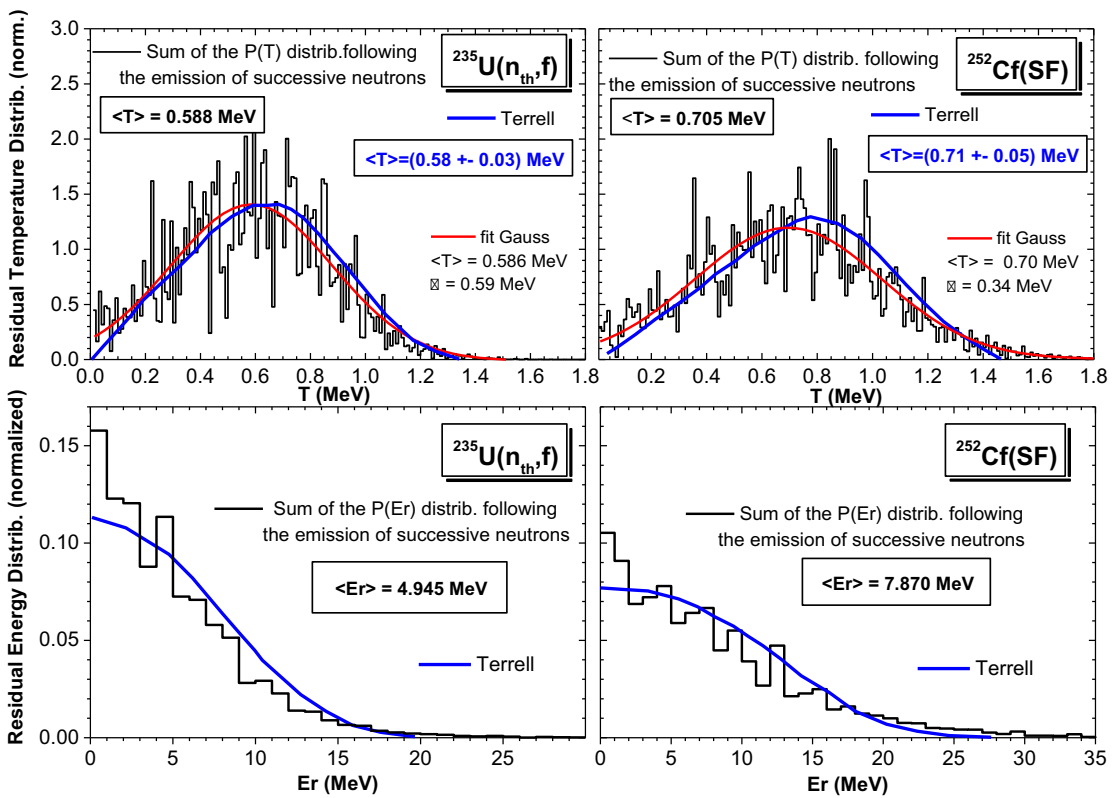

Figure 5. Sum of the residual temperature and residual energy distributions following the emission of successive neutrons from all fragments (histograms plotted black lines) in comparison with the results reported by Terrell [11] (blue lines).

(with $\mathrm{k}$ from 1 to $\mathrm{k}_{\max }(\mathrm{A}, \mathrm{Z}, \mathrm{TKE})$ ), i.e.

$$
E_{\gamma}^{(k)}(A, Z, T K E)=\bar{E}_{r}^{(k-1)}(A, Z, T K E)-<\varepsilon>_{k}(A, Z, T K E)-S_{n}^{(k-1)}(A, Z, T K E)
$$

in which $\bar{E}_{r}^{(k-1)}$ and $S_{n}^{(k-1)}$ are the average excitation energy and the neutron separation energy of the residual nucleus $\mathrm{Z}, \mathrm{A}-\mathrm{k}+1$ and $\langle\varepsilon\rangle_{k}$ is the average energy in the centre-of-mass frame of the kth emitted neutron. These quantities are obtained by solving the transcendent equation of residual temperature (1) at each sequence. The average prompt $\gamma$-ray energy corresponding to the initial fragment A, Z, TKE (i.e. the quantity expressed by Eq. (6) in the case of $\mathrm{PbP}$ ) is obtained by averaging $E_{\gamma}^{(k)}(A, Z, T K E)$ given by Eq. (7) over the number of sequential emissions according to Eq. (5).

The sum of the residual temperature and residual energy distributions following the emission of successive neutrons from all fragments are given as histograms (black lines) in Fig. 5. As it can be seen the sum of residual temperature distributions is well fitted by Gaussian functions (red lines).

Both distributions, of the residual temperature and the residual energy, are in good agreement with the results of Terrell [11] (blue lines). The average values of the residual temperature (given in each frame of Fig. 5) are very close to the average values reported by Terrell (given in blue).

The good agreement of different prompt emission quantities resulting from the sequential emission calculation with the experimental data and the results of other prompt emission models, as well as the good agreement of the sum of residual temperature and residual energy distributions following the successive neutron emission from all fragments with the results of Terrell, validate the present modelling (with the assumptions and approximations mentioned in Sect. 1). 


\section{New parameterisation of the residual temperature distribution of light and heavy fragments}

In the deterministic models Los Alamos [12,13] and PbP (Ref. [1] and references therein) the sequential emission is taken into account in a global way by a residual temperature distribution $\mathrm{P}(\mathrm{T})$ with the following form proposed by Madland and Nix [12]:

$$
P(T)= \begin{cases}\left(2 / T_{m}^{2}\right) & T \quad T \leq T_{m} \\ 0 & T>T_{m}\end{cases}
$$

In the $\mathrm{PbP}$ model the maximum temperature Tm of Eq. (8) is the temperature corresponding to the initial fragment $\mathrm{A}, \mathrm{Z}$ at a given TKE. In the LA model $T_{m}$ is the average value of the initial temperature corresponding to the light and heavy fragment groups, i.e. the LA model with non-equal Tm of Ref. [13], or it is the equivalent temperature corresponding to all fragments, i.e. the LA model with equal Tm of Ref. [12]. Consequently the prompt neutron spectrum in the centre-of-mass frame $\Phi(\varepsilon)$ corresponding to an initial fragment at a given TKE is calculated by integrating the prompt neutron spectrum at a given residual temperature $\varphi(\varepsilon, \mathrm{T})$ over the $\mathrm{P}(\mathrm{T})$ distribution.

The residual temperature distributions corresponding to the light and heavy fragment groups resulting from the modelling of sequential emission described in Sects. 2 and 3 offer the possibility to obtain a new parameterisation of $\mathrm{P}(\mathrm{T})$ as a function of the temperature of initial fragments.

Examples of $\mathrm{P}(\mathrm{T})$ distributions resulting from sequential emission calculations are illustrated in Fig. 6 (as histograms plotted with grey lines) for heavy (upper part) and light fragments (lower part).

The $\mathrm{P}(\mathrm{T})$ distributions of Madland and Nix, expressed by Eq. (8), are plotted with blue lines. The corresponding $\mathrm{Tm}$ (i.e. the average value of the initial temperature) is given in each frame.

The proposed form of $\mathrm{P}(\mathrm{T})$ is defined as:

$$
P(T)= \begin{cases}\left(P_{\max } / T_{1}\right) T & T \leq T_{1} \\ \left(P_{\max } /\left(T_{2}-T_{1}\right)\right)\left(T_{2}-T\right) & T_{1} \leq T \leq T_{2}\end{cases}
$$

with the following conditions:

i) Normalization to 1 from which the relation $P_{\max }=2 / T_{2}$ is obtained,

ii) The average $<\mathrm{T}>$ of the $\mathrm{P}(\mathrm{T})$ distribution of Eq. (7) must be equal to the $<\mathrm{T}>$ value resulting from the sequential emission calculations (which is given in each frame of Fig. 6). From this condition the relation $T_{2}=3<T>-T_{1}$ is obtained.

For the studied fissioning systems ${ }^{235} \mathrm{U}\left(\mathrm{n}_{\mathrm{th}}, \mathrm{f}\right),{ }^{239} \mathrm{Pu}\left(\mathrm{n}_{\mathrm{th}}, \mathrm{f}\right)$ and ${ }^{252} \mathrm{Cf}(\mathrm{SF})$ we have observed that the values of average residual temperatures of the light and heavy fragment groups resulting from the sequential emission calculations are approximately 0.66 from the average temperature of initial fragments, i.e. $\langle\mathrm{T}\rangle=0.66<T_{i}>$. In other words $\langle T\rangle=(2 / 3)<T_{i}>$ as in the case of $\mathrm{P}(\mathrm{T})$ of Madland and Nix. Taking into account this observation and the relations resulting from the conditions i) and ii), the following preliminary parameterisation of $\mathrm{P}(\mathrm{T})$ is proposed:

$$
P(T)= \begin{cases}\left(2 / T_{i}^{2}\right) \alpha T & T \leq c T_{i} \\ \left(2 / T_{i}^{2}\right)\left(\beta T_{i}-\gamma T\right) & c T_{i} \leq T \leq(2-c) T_{i}\end{cases}
$$

with

$$
1 / \alpha=c(2-c) ; \quad 1 / \beta=2(1-c) ; \quad 1 / \gamma=2(1-c)(2-c)
$$

In the $\mathrm{PbP}$ treatment, Ti of Eq. (10) denotes the temperature of each initial fragment $\mathrm{A}, \mathrm{Z}$ at a given TKE. In the case of the most probable fragmentation approach (i.e. the LA model with non-equal $\left.T_{m}\right) T_{i}$ is just $T_{m}$ (i.e. the average initial temperature of the light and heavy fragment group). The 

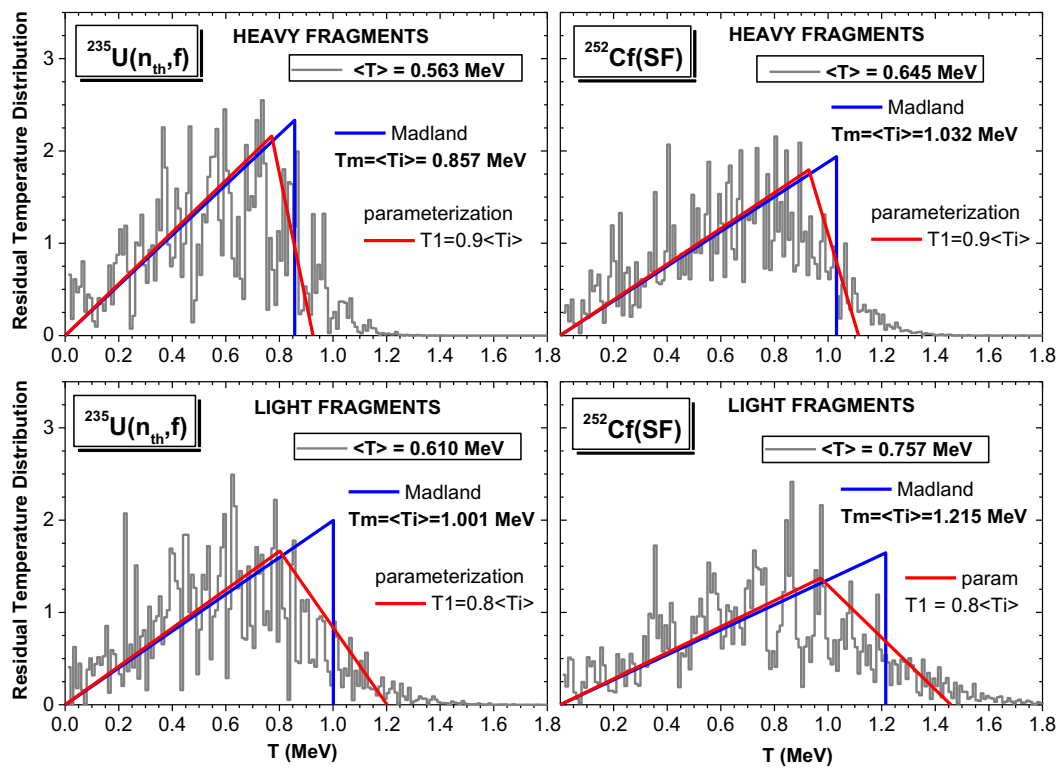

Figure 6. Examples of $\mathrm{P}(\mathrm{T})$ for the heavy (upper part) and light fragments (lower part): $\mathrm{P}(\mathrm{T})$ from sequential emission calculations (as histograms plotted with grey lines), $\mathrm{P}(\mathrm{T})$ of Madland and Nix (blue lines) and the proposed parameterisation of $\mathrm{P}(\mathrm{T})$ (red lines).

preliminary parameterisation of Eqs. $((10),(11))$ is plotted in Fig. 6 with a red line (i.e. the case $\mathrm{c}=$ 0.9 for heavy fragments and $\mathrm{c}=0.8$ for light fragments as indicated in the figure).

The use of the preliminary $\mathrm{P}(\mathrm{T})$ parameterisation of Eqs. ((8), (9)) in the PbP model calculations leads to results of the prompt neutron multiplicity and prompt $\gamma$-ray energy (e.g. $v$ (A,TKE), $v(\mathrm{~A}), v(\mathrm{TKE}),<\mathrm{E} \gamma>(\mathrm{A}),<\mathrm{E} \gamma>$ (TKE)) which do not differ significantly from the previous $\mathrm{PbP}$ results (plotted with red circles in the figures of Sect. 3) obtained with $\mathrm{P}(\mathrm{T})$ of Madland and Nix.

The improvement is seen in the prompt neutron spectrum results. An example is given in Fig. 7 where the prompt neutron spectrum in the laboratory frame of ${ }^{252} \mathrm{Cf}(\mathrm{SF})$ calculated with $\mathrm{P}(\mathrm{T})$ of Eqs. ((8), (9)) describes better the data of Poenitz and Tamura (left frame) than the calculation done with $\mathrm{P}(\mathrm{T})$ of Eq. (6) (right frame).

\section{Conclusions}

Detailed calculations taking into account the successive emission of prompt neutrons were done for three fissioning systems ${ }^{235} \mathrm{U}\left(\mathrm{n}_{\mathrm{th}}, \mathrm{f}\right),{ }^{239} \mathrm{Pu}\left(\mathrm{n}_{\mathrm{th}}, \mathrm{f}\right)$ and ${ }^{252} \mathrm{Cf}(\mathrm{SF})$. The successive transcendent equations of residual temperatures based on the assumption of fragment level densities in the Fermi-gas regime were solved under the approximations:

- non-energy dependent level density parameters of fragments (provided by the Egidy-Bucurescu systematic for BSFG) and

- an analytical expression of the compound nucleus cross-section $\sigma_{c}(\varepsilon)$ of the inverse process of neutron evaporation, which approximates well the average prompt neutron energy in the centre-ofmass frame $<\varepsilon>$ obtained with $\sigma_{c}(\varepsilon)$ provided by optical model calculations. 

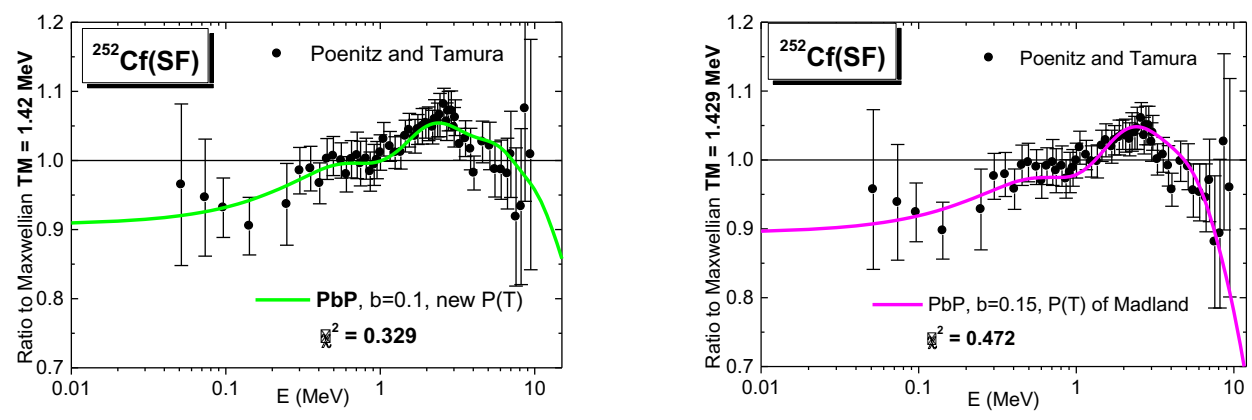

Figure 7. Prompt neutron spectrum of ${ }^{252} \mathrm{Cf}(\mathrm{SF})$ in the laboratory frame calculated with the preliminary parameterisation of $\mathrm{P}(\mathrm{T})$ (left part) and the $\mathrm{P}(\mathrm{T})$ of Madland and Nix (right part) in comparison with the data of Poenitz and Tamura (renormalized to the calculated spectrum).

This new deterministic modelling of sequential emission is validated by the good agreement of its results (e.g. $v(\mathrm{~A}), v(\mathrm{TKE}), \mathrm{E} \gamma(\mathrm{A}), \mathrm{E} \gamma(\mathrm{TKE}),<\varepsilon>(\mathrm{A}),<\varepsilon>$ (TKE)) with the experimental data and the results of other prompt emission models (e.g. PbP, FIFRELIN). Also the sum of the residual temperature and residual energy distributions following the successive neutron emission from all fragments resulting from this modelling are in good agreement with the distributions reported by Terrell [11].

The residual temperature distributions of the light and heavy fragment groups provided by this modelling allow to obtain a preliminary parameterisation of these $\mathrm{P}(\mathrm{T})$ distributions which can be used in the deterministic models PbP and Los Alamos with non-equal Tm recently proposed by Madland and Kahler [13].

The use of this preliminary $\mathrm{P}(\mathrm{T})$ parameterisation in the $\mathrm{PbP}$ model leads to prompt neutron spectrum results describing better the experimental data than the results based on the classical $\mathrm{P}(\mathrm{T})$ form of Madland and Nix [12]. The PbP results of prompt neutron multiplicity and prompt $\gamma$-ray energy (e.g. $v(\mathrm{~A}, \mathrm{TKE}), v(\mathrm{~A}), v(\mathrm{TKE}),<\mathrm{E} \gamma>(\mathrm{A}),<\mathrm{E} \gamma>(\mathrm{TKE})$ ) obtained with this preliminary parameterisation of $\mathrm{P}(\mathrm{T})$ do not differ significantly from the results obtained with $\mathrm{P}(\mathrm{T})$ of Madland and Nix.

The results reported in this paper are preliminary. Sequential emission calculations for other neutron induced fissioning nuclei at higher incident energies $\left({ }^{234,238} \mathrm{U}(\mathrm{n}, \mathrm{f}),{ }^{237} \mathrm{~Np}(\mathrm{n}, \mathrm{f})\right.$ up to about $5 \mathrm{MeV}$ ) are in progress, in order to provide an improved parameterisation of $\mathrm{P}(\mathrm{T})$. The solving of the successive transcendent equations of residual temperatures by using other prescriptions for the level density parameter of initial and residual fragments and maybe another analytical expression of $\sigma_{c}(\varepsilon)$ which will approximate better the shape of $\sigma_{c}(\varepsilon)$ provided by optical model calculations is foreseen.

\section{References}

[1] R. Capote, Chen Y.J., F.-J. Hambsch, N.V. Kornilov, J.P. Lestone, O. Litaize, B. Morillon, D. Neudecker, S. Oberstedt, T. Ohsawa, N. Otuka, V.G. Pronyaev, A. Saxena, O. Serot, O.A. Shcherbakov, Shu N.C., D.L. Smith, P. Talou, A. Trkov, A.C. Tudora, R. Vogt, S. Vorobyev, Nucl. Data Sheets 131, 1 (2016)

[2] A. Göök, F.-J. Hambsch, M. Vidali, Phys. Rev. C 90, 064611 (2014)

[3] A. Göök, F.-J. Hambsch, S. Oberstedt "Prompt neutron emission and energy balance in 235U(n,f)" ND-2016 Int. Conf. on Nuclear Data for Science and Technology, 11-16 September 2016, Bruges, Belgium

[4] A.C. Wahl, Atomic Data and Nuclear Data Tables 39, 1 (1988) 
[5] C. Morariu, A. Tudora, F.-J. Hambsch, S. Oberstedt, C. Manailescu, J. Phys. G, Nucl. Part. Phys. 39, 055103 (2012)

[6] A. Tudora, F.-J. Hambsch, I. Visan, G. Giubega, Nucl. Phys. A 940, 242 (2015)

[7] T. von Egidy and D. Bucurescu, Phys. Rev. C 80, 054310 (2009)

[8] A. Gilbert, A.C.W. Cameron, Can. J. Phys. 43, 1446 (1965)

[9] O. Iwamoto, J. Nucl. Sci. Tech. 45, 910 (2008)

[10] A. Göök, F.-J. Hambsch, S. Oberstedt "Prompt fission neutron emission in the reaction ${ }^{235} \mathrm{U}(\mathrm{n}, \mathrm{f})$ ", this workshop

[11] J. Terrell, Phys. Rev. 113, 527 (1959)

[12] D.G. Madland and J.R. Nix, Nucl. Sci. Eng. 81, 213 (1982)

[13] D.G. Madland, A.C. Kahler, Nucl. Phys. A 957, 289 (2017) 
